\title{
EXTENSION OF A KOLMOGOROV ATMOSPHERIC TURBULENCE MODEL FOR TIME-BASED SIMULATION IMPLEMENTATION
}

\author{
John D. McMinn \\ NASA Langley Research Center \\ Hampton, Va
}

\begin{abstract}
The development of any super/hypersonic aircraft requires the interaction of a wide variety of technical disciplines to maximize vehicle performance. For flight and engine control system design and development on this class of vehicle, realistic mathematical simulation models of atmospheric turbulence, including winds and the varying thermodynamic properties of the atmosphere, are needed. A model which has been tentatively selected by a government/industry group of flight and engine/inlet controls representatives working on the High Speed Civil Transport is one based on the Kolmogorov spectrum function. This report compares the Dryden and Kolmogorov turbulence forms, and describes enhancements that add functionality to the selected Kolmogorov model. These added features are: an altitude variation of the eddy dissipation rate based on Dryden data, the mapping of the eddy dissipation rate database onto a regular latitude and longitude grid, a method to account for flight at large vehicle attitude angles, and a procedure for transitioning smoothly across turbulence segments.

Symbols

$\mathrm{A}_{\mathrm{n}} \quad$ Fourier series cosine coefficient

$B_{n} \quad$ Fourier series sine coefficient

E Kolmogorov energy spectrum

$f_{1} \quad$ lower bound on frequency range of interest, $\mathrm{Hz}$

$f_{2}$ upper bound on frequency range of interest, $\mathrm{Hz}$

h altitude, $\mathrm{km}$

$k_{1} \quad$ lower bound on wavenumber range of interest, $\mathrm{rad} / \mathrm{m}$

$k_{2}$ upper bound on wavenumber range of interest, $\mathrm{rad} / \mathrm{m}$

$\mathrm{L} \quad$ integral scale length, $m$

$\mathrm{L}_{0} \quad$ Kolmogorov outer scale length, $m$

u longitudinal turbulence velocity, $\mathrm{m} / \mathrm{s}$

v transverse turbulence velocity component, $\mathrm{m} / \mathrm{s}$
\end{abstract}

\footnotetext{
'Aerospace Engineer, Flight Dynamics and Control Division, Member AIAA

Copyright 11997 by the American Institute of Aeronautics and Astronautics, Inc. No copyright is asserted in the United States under Title 17, U.S. Code. The U.S. Government has a royalty-free license to exercise all rights under the copyright claimed herein for Governmental Purposes. All other rights are reserved by the copyright owner.
}

V air relative vehicle velocity, excluding turbulence components, $\mathrm{m} / \mathrm{s}$

w turbulence velocity component orthogonal to $u$ and $\mathrm{v}, \mathrm{m} / \mathrm{s}$

$x$ distance traveled along flight path, $m$

$\alpha \quad$ general Kolmogorov energy spectrum constant

$\alpha_{p} \quad$ Kolmogorov pressure energy spectrum constant, $\mathrm{hPa}^{2} \mathrm{~s}^{2} / \mathrm{m}^{2}$

$\gamma \quad$ flight path angle, deg

$\Delta p \quad$ density perturbation from mean

$\Delta \mathrm{P} \quad$ pressure perturbation from mean

$\Delta \mathrm{T}$ temperature perturbation from mean

$\varepsilon \quad$ eddy dissipation rate, $\mathrm{m}^{2} / \mathrm{s}^{3}$

$\varepsilon_{\mathrm{D}} \quad$ eddy dissipation rate derived from Dryden data, $\mathrm{m}^{2} / \mathrm{s}^{3}$

$\varepsilon_{0} \quad$ eddy dissipation rate used in $A_{n}$ and $B_{n}$ calculation, $\mathrm{m}^{2} / \mathrm{s}^{3}$

$\Phi \quad$ Dryden energy spectrum

$\Phi_{v} \quad$ von Karman energy spectrum

$\phi \quad$ body axis roll angle, deg

$\theta$ pitch attitude, deg

$\Psi_{w} \quad$ heading relative to wind, $\mathrm{deg}$

$\Omega \quad$ wavenumber, $\mathrm{rad} / \mathrm{m}$

$\sigma \quad$ RMS turbulence velocity, $\mathrm{m} / \mathrm{s}$

\section{Abbreviations}

3-D three dimensional

2-D two dimensional

lat. latitude

long. longitude

HSCT High Speed Civil Transport

RMS Root Mean Square

\begin{tabular}{ll}
\multicolumn{2}{l}{ Subscripts } \\
$\mathrm{h}$ & horizontal component \\
$\mathrm{u}$ & longitudinal component \\
$\mathrm{v}$ & transverse component \\
$\mathrm{w}$ & component orthogonal to $\mathrm{u}$ and $\mathrm{v}$ \\
$\mathrm{O}$ & indicates freestream value
\end{tabular}

\section{Introduction}

The development of any new aircraft requires the interaction of a wide variety of technical disciplines. Furthermore, when the vehicle being designed requires 
the development of new technology in many of these disciplines, the level of interaction between the technology teams must be high to maximize efficiency in the resulting design. Such is the case with the technology development of the High Speed Civil Transport (HSCT) under the High Speed Research program.

In the case of the HSCT program it was recognized that different disciplines used different atmosphere models. In order to establish a common basis for comparison and minimize duplication of effort, an ad hoc group, including representatives from NASA and industry, has formed with the goal of adopting a model of atmospheric turbulence. The selected model will be used within the engine community as a basis for the evaluation of inlet/engine performance and in the flight controls area for the evaluation of the aircraft control system and handling qualities in piloted and batch computer simulations.

The model which has been proposed by the group is one suggested by Bill Tank of Boeing'. The model, like the von Karman model, is defined in the frequency domain, and for which the power has a $-5 / 3$ intensity roll off with increasing frequency. A key reason for the selection of the model is the capability to generate thermodynamic perturbations (density, pressure, and temperature) as well as wind gusts. A second reason for selecting the Tank model is the data used to parameterize it. Tank bases the model on data collected from a variety of sources where data was taken in flight as close as possible to the approximate cruise altitude of the HSCT.

This report reviews the Tank model, discusses implementation issues, and presents extensions made to the model to enhance its capability.

\section{Background}

The atmosphere has long been studied to better understand and predict its behavior, and for aircraft designers and operators, understanding the turbulent nature of the atmosphere is an area of concern. Current atmospheric theory ${ }^{2}$ states that the turbulent energy of the atmosphere is distributed as a continuous function of wavenumber (spatial frequency) as shown in Figure 1. This Figure shows the zonal (east-west) wind, meridional (north-south) wind, and potential temperature power spectra (note: for clarity the meridional and temperature spectra have been shifted one and two decades to the right respectively). Notice that at the very long (planetary scale) wavelengths the power of turbulence decreases as the -3 power of decreasing wavelength (increasing frequency), and from about a wavelength of $500 \mathrm{~km}$, the power fall off is roughly as the $-5 / 3$ power of frequency. Turbulence theory also states that the range where turbulence is fully three dimensional and isotropic extends from the very small scale $(<1 \mathrm{~cm})$ out to a scale of a few hundred meters ${ }^{3}$.

In the range between a few hundred meters and $500 \mathrm{~km}$ the $-5 / 3$ law is still obeyed for turbulence in the near horizontal plane, though the turbulence cannot be classified as three dimensional (3-D) isotropic. Vertical turbulence has been found to obey a power fall off of -3 to -2 down to scales of 100 meters. It is expected to change to a $-5 / 3$ slope at even shorter scales ${ }^{3}$.

In the 3-D region turbulence energy is not so much lost due to friction as it is transferred to shorter and shorter wavelengths. The rate at which energy is cascaded down in scale is called the eddy dissipation rate, $\varepsilon$. There is also evidence that supports the theory that energy is not only cascaded down in scale but also up. That the $-5 / 3$ law holds for scales out to hundreds of kilometers is a fortunate result of upscale transfer of two dimensional (2-D) turbulence energy. The impact this has on the modeling of turbulence for simulations is that models developed for 3-D, isotropic turbulence at short scales can be extended beyond the 3-D regime into the margins of the 2-D without changing the model.

In order to evaluate the performance of aircraft systems and flying qualities an appropriate, relatively simple, characterization of atmospheric turbulence is required. It is not sufficient to always assume a quiescent atmosphere or even that the thermodynamic properties of the atmosphere are solely deterministic functions of altitude.

Traditionally the Dryden turbulence model has been used whenever atmospheric (wind) turbulence was needed to add some sense of realism to a time-based simulation or to provide a disturbance input when evaluating systems in the frequency domain. The Dryden spectrum for the longitudinal, body axis velocity disturbance is given ${ }^{4}$ as,

$$
\Phi(\Omega)_{u}=\sigma^{2} \frac{2 L_{u}}{\pi} \frac{1}{1+\left(L_{u} \Omega\right)^{2}}
$$

where $\Omega$ is the wavenumber, or frequency term, in units of radians per meter, $\sigma$ represents the standard deviation of the velocity disturbance, and $L_{u}$ is the integral scale of the longitudinal turbulence, which will be discussed in more detail later.

The spectra of the lateral and vertical components of Dryden body axis turbulence are given in the same reference by, 


$$
\Phi(\Omega)_{v, w}=\sigma^{2} \frac{L_{v, w}}{\pi} \frac{1+3\left(L_{v, w} \Omega\right)^{2}}{\left[1+\left(L_{v, w} \Omega\right)^{2}\right]^{2}}
$$

with a similar definition of terms. Although the Dryden equations are more frequently used, they are not the best fit to the observed character of the atmosphere. Notice in equations 1 and 2, that the Dryden spectra fall off as the -2 power of frequency, not the $-5 / 3$ power as shown in Figure 1. The predominant use of the Dryden form stems from the ease of implementation within a time-based simulation. Such implementation usually involves forming a band limited, white noise filter equation by taking the spectral square roots of the spectrum equations. This is not so simple for an irrational $-5 / 3$ power fall off without approximating, but is relatively simple for the rational -2 power fall in equations 1 and 2 . The resulting transfer functions ${ }^{4}$ are given below,

longitudinal:

$$
F_{u}(s)=\sigma_{u} \sqrt{\frac{2 V}{\pi L_{u}}}\left(\frac{1}{s+\frac{V}{L_{u}}}\right)
$$

lateral and vertical:

$$
F_{v, w}(s)=\sigma_{v, w} \sqrt{\frac{3 V}{\pi L_{v, w}}}\left(\frac{s+\frac{V}{\sqrt{3} L_{v, w}}}{\left(s+\frac{V}{L_{v, w}}\right)^{2}}\right)
$$

Use of these equations with a band-limited, unit variance, white noise input and appropriate filter parameters yields Dryden aircraft-scale turbulence. For the purpose of classical rigid-body simulation and piloted assessment, the Dryden form is sufficient. When aircraft flexibility is an issue for vehicle flight assessment the frequency range of interest widens to include the higher frequency structural modes. At this point the difference between the -2 and the $-5 / 3$ power fall off can not be ignored.

The spectrum functions that better fit experimentally observed turbulence are the von Karman spectra as given in the same reference ${ }^{4}$,

$$
\Phi_{v}(\Omega)_{u}=\sigma^{2} \frac{2 L_{u}}{\pi} \frac{1}{\left[1+\left(1.339 L_{u} \Omega\right)^{2}\right]^{\frac{5}{6}}}
$$

for the longitudinal, and
$\Phi_{v}(\Omega)_{v, w}=\sigma^{2} \frac{L_{v, w}}{\pi} \frac{1+\frac{8}{3}\left(1.339 L_{v, w} \Omega\right)^{2}}{\left[1+\left(1.339 L_{v, w} \Omega\right)^{2}\right]^{\frac{11}{6}}}$

for the lateral and vertical components.

Both the Dryden form and the von Karman are parameterized by two terms, the standard deviation of the turbulence, $\sigma$, and an integral scale length. The standard deviation is equal in magnitude to the square root of the area under a power spectrum plot. Note that increasing or decreasing $\sigma$ in the equations $1,2,5$, or 6 would have the effect of shifting up or down the amplitude of the spectrum, without distorting the shape of the plot. The Dryden spectra shown in Figure 2 were generated with the equation,

$$
\Phi(\Omega)=\frac{10}{1+(0.5 \Omega)^{2}}
$$

Referring to eqn. 1 , this equation arises when $L_{u}=0.5 \mathrm{~m}$ and $\sigma^{2}=10 \pi \mathrm{m}^{2} / \mathrm{s}^{2}$. The solid line in the upper plot on Figure 2 shows the typical Dryden spectral shape on a $\log -\log$ plot. Note that the $\log -\log$ plot is deceptive when attempting to characterize the area distribution by visual inspection. Although most of the area, and thus larger contributor to the integral of the spectrum, appears to lie under the flat (low frequency) portion of the power spectrum, that relation is not correct. As decade by decade integration of the spectrum would reveal, most of the turbulence power being described by the plot, is under the sloping portion of the curve.

The upper plot is a log-log plot and the lower a semilog of the same data, with the solid lines defined by equation 7. The dashed line in Figure 2 represents the product of the power (or Dryden amplitude) and the axis frequency. Plotting this product as a function frequency gives a more realistic (less deceptive) graphical representation of the area distribution.

The knee, or the point where the spectrum has fallen to the half power point with increasing wavenumber (or frequency), is located at a wavenumber of $1 / \mathrm{L}$. In equation 7, $\mathrm{L}$ has a value of 0.5 . As shown in Figure 2 , the knee is properly located at a wavenumber of $1 / L_{u}$, or $2.0 \mathrm{Rad} . / \mathrm{m}$. For proper use, the wavenumbers of interest to the designer should be near $1 / \mathrm{L}_{u}$, so that the power associated with those wavenumbers is appropriate. Arbitrarily increasing the scale length, $L$, has the effect of shifting the knee of the spectrum to the left. If the knee moves left and $\sigma$ is held constant, the spectrum will be shifted left and upward slightly to keep the integrated area constant. This shifting reduces the power associated with the higher frequencies. In order to both not affect the higher frequency power distribution 
and move the knee to the left (for example, to include more low frequency power), both $L$ and $\sigma$ must . increase. So it should seem intuitive now that the standard deviation and scale length should be related. Tables defining $L$ and $\sigma$ pairs, exist and give the pairs as a function of altitude, such as is shown in Table $1^{5}$.

Despite being frequently used there is much controversy over specification of the scale length. According to Etkin, the determination of the scale length at altitude remains an unresolved difficulty. This stems from the uncertainty of how to determine $\mathrm{L}$, whether from the integral of the velocity autocorrelation function or from a fit to a spectrum ${ }^{6}$. The matter is further confused by the tendency to think of the integral scale as a physical characteristic of the turbulence field, and is often confused with the 'outer scale' of turbulence, or the longest wavelength that could be expected. For example, in Figure 2 the integral scale is the reciprocal of the knee frequency, or 0.5 , but inspection of the bell shaped area distribution indicates that significant turbulence intensity exists at longer scales (lower frequencies).

Additionally, the use of the Dryden or von Karman models provide turbulent winds, but not thermodynamic variations. The models do not usually account for the global variation of turbulence. In time-based simulations, where the Dryden model is used, the power fall off is not the observed $-5 / 3$ value, though some may argue that the Dryden model gives a better fit to the vertical gusts due their observed -3 to -2 character at scales above $100 \mathrm{~m}$ as mentioned above.

\section{The Tank Turbulence Model}

In the light of these issues with using the Dryden and von Karman models, it is interesting to note that atmospheric scientists use a different model. Where the aerodynamicist uses the Dryden or von Karman models for characterizations of the turbulent atmosphere, atmospheric scientists tend to use the Kolmogorov energy spectrum ${ }^{7}$. The Kolmogorov spectrum function for longitudinal turbulence has the form,

$$
E(k)=\alpha \varepsilon^{\frac{2}{3}} k^{\frac{-5}{3}}
$$

where $k$ is the spatial wavenumber, $\varepsilon$ is the eddy dissipation rate, and $\alpha$ is a specified constant. In log space eqn. 8 is the equation for a straight line with a $-5 / 3$ slope. Notice that it matches the $-5 / 3$ character observed in Figure 1, and is similar to the von Karman form in the limit as wavenumber approaches infinity. In the 3-D range the transverse spectra are $4 / 3$ times the longitudinal for an incompressible fluid as Kolmogorov showed ${ }^{8}$.
The turbulence model suggested by Tank' for application to the HSCT, hereafter referred to as the Tank model, uses this Kolmogorov form for defining wind and thermodynamic turbulence. According to the Tank model the temperature spectrum has the same form as the longitudinal, the only difference being the constant $\alpha$, whose units vary depending on the spectrum being defined. A description of the similarity of the temperature and velocity spectra is available? The spectrum of atmospheric pressure perturbations associated with turbulence is given by Tank to be

$$
E(k)=\alpha_{p}\left(P_{0} / T_{0}\right)^{2} \varepsilon^{2 / 3} k^{-5 / 3}
$$

where $\mathrm{P}_{0}$ is the standard atmospheric pressure in $\mathrm{hPa}$ and $T_{0}$ is the standard Kelvin temperature.

The Tank model defines the temperature and pressure equations, leaving the the density perturbation to be determined from the equation of state. If,

$$
\left(P_{0}+\Delta P\right)=\left(\rho_{0}+\Delta \rho\right) R\left(T_{0}+\Delta T\right)
$$

where $\rho_{0}$ is the standard atmospheric density, $R$ is the gas constant, and the $\Delta$ symbol indicates a perturbation of an atmospheric quantity (temperature, T, or pressure, P), then the density perturbation can be solved for

$$
\Delta \rho=\frac{\left(P_{0}+\Delta P\right)}{\left(T_{0}+\Delta T\right) R}-\frac{P_{0}}{R T_{0}}
$$

Given these equations and the appropriate $\alpha$ value for the quantity being simulated, the Tank model reduces to one parameter, $\varepsilon$. This contrasts with conventional Dryden and von Karman which have two parameters ( $\sigma$ and $L$ ). The eddy dissipation rate, $\varepsilon$, characterizes the turbulence for whatever range of frequencies is of interest. To account for the global non-homogeneous nature of turbulence, Tank has defined $\varepsilon$, at the approximate HSCT cruise altitude, as shown in Figure 3. Inspection of the figure reveals that Tank has defined $\varepsilon$ between 80 degrees north and 40 degrees south latitude over the oceans around the world.

The eddy dissipation rate can be linked to more familiar terms by integrating both sides of eqn. 8 from $k_{1}$ to $k_{2}$ and recognizing that the integral of the left side is equal to $\sigma^{2}$. This yields the following equation,

$$
\varepsilon=\left(\frac{2 \sigma^{2}}{3 \alpha\left(\mathrm{k}_{2}^{\frac{-2}{3}}+\mathrm{k}_{1}^{\frac{-2}{3}}\right)}\right)^{3 / 2}
$$


Here $\sigma^{2}$ represents the area under the spectrum defined in eqn. 8 and is the variance of the turbulence within the range $k_{1}$ to $k_{2}$. Integrating from $k_{1}$ to infinite wavenumber equation 12 reduces to

$$
\varepsilon=\frac{\sigma^{3}}{(1.5 \alpha)^{\frac{3}{2}} \mathrm{~L}_{0}}
$$

where $L_{0}$ is $1 / k_{1}$ and represents the longest wavelength in the range of interest and is referred to as the outer scale length. This outer scale should not be confused with the integral scale length.

\section{Tank Model Extensions}

To implement and add functionality to a time-based simulation employing the Tank model, several features have been developed by the author in a series of MATLAB scripts. A variation of the eddy dissipation rate with altitude based on Dryden data has been calculated. The $\varepsilon$ database has been modified. Methods to more properly account for flight at large attitude angles have been implemented. Also, a procedure for transitioning smoothly from one turbulence segment to the next has been developed.

\section{Altitude Variation}

The model proposed by Tank defined the eddy dissipation rate only at the estimated cruise altitude $(\sim 60 \mathrm{kft})$ as shown in Figure 3 . To use the model in a broader range of the flight envelope a method for changing $\varepsilon$ based on altitude was developed using eqn. 13 and data from reference 3 .

Table $1^{5}$ defines a set of standard deviations and integral scale lengths for light, moderate, and severe turbulence as a function of altitude for use in a Dryden turbulence model. Using this $\sigma$ and $L$ information for horizontal turbulence, with $\alpha$ (in eqn. 13) set equal to 0.15 as specified by Tank for longitudinal turbulence, eddy dissipation rates were estimated from the Dryden parameters. These estimated eddy dissipation rates are shown in Table 2.

Noting that Tank defines the lower stratosphere global mean epsilon to $0.00002 \mathrm{~m}^{2} / \mathrm{s}^{3}$ and observing that at an altitude of $18 \mathrm{~km}$ the light, Dryden turbulence data produced a nearly identical epsilon value (shaded in Table 2), it was accepted that the 'light' turbulence data best fit Tank's data. To support that claim, two time histories were evolved, one by the Tank method and the other with the Dryden filter. Sample time histories can be compared in Figure 4 and the corresponding power spectra in Figure 5. Notice that both models generate nearly the same low frequency character and that this observation is substantiated by the overlap of the power spectra at the low frequencies. Also note that the Tank model produces the higher amplitude high frequency turbulence, this is due to the difference in power fall off between the two models. As should be expected by looking at the power spectra the standard deviation of the Tank time history is higher than the Dryden (.2699 vs. $.2158 \mathrm{~m} / \mathrm{s}$ ).

Accepting the similarity, the light turbulence $\varepsilon$ data was normalized to unity at $18 \mathrm{~km}$ and is shown in the last column of Table 2 . The resulting normalized variation with altitude is the altitude variation that has been added through eqn. 14,

$\varepsilon$ (lat., long., $h)=\varepsilon$ (lat., long.) $* \varepsilon_{D}(h)$

where $h$ is altitude in $\mathrm{km}$.

\section{Database Gridding}

The $\varepsilon$ data given by Tank $^{1}$ was only presented in the form of the isoline plot shown as Figure 3. For implementation within a computer simulation, the data was mapped into a regular latitude and longitude grid as shown in Table 3. The missing data over the continents was filled in with a constant value of .000021 corresponding to the global average $\varepsilon$ value of .00002 plus an offset (the insignificant offset was used for bookkeeping purposes to identify the value as one not originally defined by Tank).

\section{Large Angle Flight}

To explain the remainder of the additions to the Tank model first requires the explanation of the time-based implementation method.

With the spectrum defined in eqn. 8, any of a number of methods could be used to evolve a time simulation.

Tank suggests a method described by Fairall, White, and Thompson ${ }^{3}$. If the spectrum is discretized in $\mathbf{M}$ frequency bands $\Delta k$ wide between $k_{1}$ and $k_{2}$, the strip area can be computed and equated as

$$
\text { area }=0.5 *\left(\mathrm{~A}_{n}{ }^{2}+\mathrm{B}_{n}{ }^{2}\right)
$$

where $\Delta k$ is defined as numerically equal to $k_{1}$ (the lowest frequency of interest), and $n$ ranges from 1 to $M$.

After solving for $A_{n}$ and $B_{n}$, with a uniform random split between $A_{n}$ and $B_{n}$, a Fourier series can be used to represent a turbulence field as follows

$$
u(x)=\sum_{n=1}^{M} A_{n} \cos \left(\frac{2 \pi x n}{L_{0}}\right)+B_{n} \sin \left(\frac{2 \pi \times n}{L_{0}}\right)
$$

Here $L_{0}$ is the segment length $1 / k_{1}$. When the sequence above is evolved a one dimensional turbulence field in 
space is defined. Using Taylor's frozen field hypothesis the space field can be converted to a time-based sequence with the simple transformation

$$
\mathrm{x}=\mathrm{V} * \mathrm{t}
$$

Incorporating this and exchanging $\Delta k$ for $1 / L_{0}$, eqn. 16 becomes

$$
u(t)=\sum_{n=1}^{M} A_{n} \cos (2 \pi \Delta k t n)+B_{n} \sin (2 \pi \Delta k t n)
$$

If evolved for a time longer than it takes to travel a distance $L_{0}$, as defined by eqn. 17 , the field will begin to repeat. To avoid this a new set of $A_{n}$ and $B_{n}$ coefficients must be computed and used each time a distance $L_{0}$ is traversed.

Given this method of generating a turbulence field, several comments can be made. The frequency range of interest is defined in terms of spatial waves (if the vehicle velocity changes, the effective frequency changes). The turbulence field is evolved in precomputed segments. The vehicle state does not continuously update the model. The vehicle orientation has no effect on the sensed winds.

\section{Dissipation Rate Scaling}

To account for the fact that $\varepsilon$ may change significantly during a segment that maybe as long as $100 \mathrm{~km}, \varepsilon$ scaling has been implemented. After computing the turbulence field with a fixed eddy dissipation rate, $\varepsilon_{0,}$, the instantaneous value of $\varepsilon$ can be used to form a scaling term,

$$
\text { scale factor }=\left(\varepsilon / \varepsilon_{0}\right)^{1 / 3}
$$

Equation 19 was derived through algebraic manipulation of equation 15 (with the strip area equal to the integral of equation 8) and observing the effect of $\varepsilon$ on $A_{n}$ and $B_{n}$. This scaling factor is applied directly to all the turbulence quantities. Figure 6 contains two traces of temperature as a function of time. The upper trace shows dissipation rate scaling as $\varepsilon$ is arbitrarily increased by a factor of 4 over the 250 second run. The lower trace, which has been shifted down 2 degrees for clarity, is the same sequence without scaling. Notice how the magnitude of the perturbations increases in the upper trace and not in the lower.

\section{Coordinate System Rotation}

As in the case of Dryden turbulence, the Tank model as defined produces body axis turbulence for horizontally flying vehicles. This restriction is the result of the turbulence filters being derived for a turbulence field aligned along and normal to the vehicle velocity vector. Also, for horizontal flight, the vehicle altitude is nearly constant so the filter parameters, which are usually functions of altitude, do not change. To allow simulation of flight at large vehicle attitude angles, where there may be significant misalignment of the velocity vector and $x$-body axis, or large roll angle, a transformation has been defined.

To develop the axis transformation equations, the axis systems had to be defined. For ease of implementation it was desired that the output of the turbulence model should be body axis turbulence. It was also known that the longitudinal turbulence equation was specifically derived to generate turbulence along the velocity vector. The two remaining axes are only required to be normal to the longitudinal (and each other). For simplicity, in this implementation, one of these axes always lies in the local horizontal. The third axis points as required to remain normal to the local horizontal and the velocity vector. This choice has the benefit of aligning with the gravity and body axes in the case of standard horizontal flight.

To compute the transformation the heading 'off the wind' $\left(\Psi_{w}\right)$, pitch attitude $(\theta)$, flight path angle $(\gamma)$, and roll angle $(\phi)$ are required. Rotating from these turbulence axes to body axes involves first a rotation through $\Psi_{w}$ about the local vertical axis. Second, is an upward rotation about the one axis lying in the local horizontal plane that is normal to the velocity vector, through the angle $\theta-\gamma$. The final rotation is about the $\mathrm{x}$-body axis through the roll angle $\phi$.

The turbulence to body axis transformation matrix can be written as the reverse order multiplication of the individual vector rotations as follows

$$
\begin{aligned}
& \mathrm{T}_{\mathrm{BT}}= \\
& {\left[\begin{array}{ccc}
1 & 0 & 0 \\
0 & \cos \phi & \sin \phi \\
0 & -\sin \phi & \cos \phi
\end{array}\right]\left[\begin{array}{ccc}
\cos (\theta-\gamma) & 0 & -\sin (\theta-\gamma) \\
0 & 1 & 0 \\
\sin (\theta-\gamma) & 0 & \cos (\theta-\gamma)
\end{array}\right]\left[\begin{array}{ccc}
\cos \Psi_{\mathrm{w}} & \sin \Psi_{\mathrm{w}} & 0 \\
-\sin \Psi_{\mathrm{w}} & \cos \Psi_{\mathrm{w}} & 0 \\
0 & 0 & 1
\end{array}\right]}
\end{aligned}
$$

At each time step then the turbulence velocities are rotated into body axes with the following equation,

$$
\left[\begin{array}{c}
\mathrm{u} \\
\mathrm{v} \\
\mathrm{w}
\end{array}\right]_{\text {body }}=\left[\mathrm{T}_{\mathrm{BT}}\left[\begin{array}{c}
\mathrm{u} \\
\mathrm{v} \\
\mathrm{w}
\end{array}\right]_{\text {turbulence }}\right.
$$

Figure 7 shows the effect of the transformation in the case of a complete roll about the longitudinal body axis. The roll maneuver begins at the 10 second mark and is completed in 24 seconds. The top two traces are the 
turbulence time histories of the lateral components as generated in the turbulence axis system. The bottom two traces are the corresponding plots in body axes. Notice that the corresponding $v$ and $w$ plots are identical for the first and last 10 seconds in the traces. Between 10 and 34 seconds the roll occurs. Notice at the half way point the two $\mathrm{v}$ plots and two $\mathrm{w}$ plots are equal in magnitude but opposite in sign, as they should be for 180 degrees of roll.

\section{Turbulence Segments}

The model as described by Tank was developed for simulations with the simulation time duration defined a priori. The low frequency wave number was then computed as the quotient of the time duration and the flight velocity. This manner of implementation allows one set of Fourier coefficients to generate the entire turbulence field.

When the simulation time duration is not known, the low frequency wavenumber must be specified. If the duration of the simulation is long enough for the vehicle to travel a distance greater then the reciprocal of the selected low frequency wavenumber, hereafter called the segment distance, the turbulence field would begin to repeat. To avoid the repeating pattern, a new set of Fourier coefficients must be calculated each time a segment ends.

This changing of Fourier coefficients allows the likely possibility that a significant discontinuity could exist between segments. To minimize the discontinuity, an exponential decay from the last value of the previous segment is faired into the following segment according to,

$$
u(t)=u^{\prime}(t)+(\Delta u) \exp \left(\frac{f_{2}}{2} \Delta t\right)
$$

where $u^{\prime}(t)$ is the raw turbulence perturbation as calculated, $\Delta \mathbf{u}$ is the magnitude of the discontinuity between the segments, $f_{2}$ is the upper frequency limit, and $\Delta t$ in this instance is the time since the discontinuity. The result of equation 23 is that the discontinuous jump is faired in over a few seconds in most cases. Figure 8 shows a turbulence time history sequence in its raw and faired form. In this example the minimum frequency of interest was $0.05 \mathrm{~Hz}$, therefore a discontinuity occurs every 20 seconds. In Figure 8 they are visible in the raw sequence (dotted line) at the 260 and 280 second points. Notice that the faired sequence (solid line) does not follow the discontinuity, but quickly merges with the raw sequence shortly thereafter.

\section{Summary}

In this report Dryden and Kolmogorov based turbulence models have been reviewed with attention paid to the parameterization of the models. The specific Kolmogorov model proposed by Tank has been implemented as a series of MATLAB scripts. Features that add functionality to the Tank model have been developed, implemented and described. A variation of the eddy dissipation rate with altitude based on Dryden data has been calculated. The eddy dissipation rate database has been mapped onto a regular latitude and longitude grid and over-land values filled in. Methods to account for flight at large vehicle attitude angles have been implemented. Finally, a procedure for transitioning smoothly across turbulence segments has been developed.

\section{References}

1. Tank, William G.; Atmospheric Disturbance Environment Definition, NASA CR-195315, February 1994.

2. Nastrom, G.D.; Gage, K.S.: A Climatology of Atmospheric Wavenumber Spectra of Wind and Temperature Observed by Commercial Aircraft. J. Atmospheric Sciences, vol. 42, No. 9, May 1985, pp. 950-960.

3. Fairall, C.W.; White, A.B.; Thompson, Dennis W.; A Stochastic Model of Gravity-Wave-Induced Clear-Air Turbulence, J. Atmos. Sci. Vol. 48, No. 15, August, 1991.

4. Hoblit, Frederic M.; Gust Loads on Aircraft: Concepts and Applications, AIAA, Washington DC, 1988

5. Johnson, D. L.; Terrestrial Environment (Climatic) Criteria for Use in Aerospace Vehicle Development, 1993 Revision; NASA TM 4511, August 1993.

6. Etkin, B.; Turbulent Wind and Its Effect on Flight, J. Aircraft Vol. 18, No. 5, May 1981.

7. Cornman, L.; Morse, C.; Cunning, G.; Real-time Estimation of Atmospheric Turbulence Severity From In-Situ Aircraft Measurements, AIAA 94-0268, 32nd Aerospace Sciences Meeting \& Exhibit, Reno NV, January, 1994.

8.Gage, K.S.: Evidence for a k-5/3 Law Inertial Range in Mesoscale Two-Dimensional Turbulence. J. Atmospheric Sciences, vol. 36, October 1979, pp. 1350-1354.

9. Tennekes, H.; Lumley, J.L.; A First Course In Turbulence, The MIT Press, Cambridge 1972 
Table 1. Dryden horizontal and vertical turbulence parameters as functions of altitude and severity.

\begin{tabular}{|c|c|c|c|c|c|c|c|c|}
\hline & \multicolumn{2}{|c|}{ Light Turbulence } & \multicolumn{2}{|c|}{ Moderate Turb. } & \multicolumn{2}{|c|}{ Severe Turbulence } & \multicolumn{2}{|c|}{ Scale Lengths } \\
\hline $\begin{array}{c}\text { Altitude } \\
\text { km }\end{array}$ & $\begin{array}{c}\text { Horizontal } \\
\sigma_{\mathrm{h}} \\
\mathrm{m} / \mathrm{s}\end{array}$ & $\begin{array}{c}\text { Vertical } \\
\sigma_{\mathrm{v}, \mathrm{w}} \\
\mathrm{m} / \mathrm{s} \\
\end{array}$ & $\begin{array}{c}\text { Horizontal } \\
\sigma_{\mathrm{h}} \\
\mathrm{m} / \mathrm{s}\end{array}$ & $\begin{array}{l}\text { Vertical } \\
\sigma_{\mathrm{v}, \mathrm{w}} \\
\mathrm{m} / \mathrm{s} \\
\end{array}$ & $\begin{array}{c}\text { Horizontal } \\
\sigma_{\mathrm{h}} \\
\mathrm{m} / \mathrm{s}\end{array}$ & $\begin{array}{c}\text { Vertical } \\
\sigma_{\mathrm{v}, \mathrm{w}} \\
\mathrm{m} / \mathrm{s}\end{array}$ & $\begin{array}{c}\text { Horizontal } \\
\mathrm{L}_{\mathrm{u}} \\
\mathrm{km}\end{array}$ & $\begin{array}{c}\text { Vertical } \\
\mathbf{L}_{\mathrm{v}, \mathrm{w}} \\
\mathbf{k m}\end{array}$ \\
\hline 1 & 0.17 & 0.14 & 1.65 & 1.36 & 5.70 & 4.67 & 0.832 & 0.624 \\
\hline 2 & 0.17 & 0.14 & 1.65 & 1.43 & 5.80 & 4.75 & 0.902 & 0.831 \\
\hline 4 & 0.20 & 0.17 & 2.04 & 1.68 & 6.24 & 5.13 & 1.04 & 0.972 \\
\hline 6 & 0.21 & 0.17 & 2.13 & 1.69 & 7.16 & 5.69 & 1.04 & 1.01 \\
\hline 8 & 0.22 & 0.17 & 2.15 & 1.69 & 7.59 & 5.98 & 1.04 & 0.98 \\
\hline 10 & 0.22 & 0.17 & 2.23 & 1.73 & 7.72 & 6.00 & 1.23 & 1.10 \\
\hline 12 & 0.25 & 0.18 & 2.47 & 1.79 & 7.89 & 5.71 & 1.80 & 1.54 \\
\hline 14 & 0.26 & 0.19 & 2.62 & 1.91 & 6.93 & 5.05 & 2.82 & 2.12 \\
\hline 16 & 0.24 & 0.21 & 2.44 & 2.10 & 5.00 & 4.31 & 3.40 & 2.6 \\
\hline 18 & 0.22 & 0.21 & 2.21 & 2.07 & 4.07 & 3.81 & 5.00 & 3.34 \\
\hline 20 & 0.23 & 0.20 & 2.26 & 1.99 & 3.85 & 3.38 & 8.64 & 4.41 \\
\hline 25 & 0.27 & 0.21 & 2.71 & 2.09 & 4.34 & 3.34 & 12 & 6.56 \\
\hline 30 & 0.37 & 0.24 & 3.73 & 2.39 & 5.6 & 3.59 & 28.6 & 8.88 \\
\hline
\end{tabular}

Table 2. Epsilon values derived from Table 1 data.

\begin{tabular}{|c|c|c|c|c|}
\hline $\begin{array}{c}\text { Altitude, } \\
\mathrm{km}\end{array}$ & $\begin{array}{c}\text { Light Turbulence } \\
\varepsilon, \mathrm{m}^{2} / \mathrm{s}^{3}\end{array}$ & $\begin{array}{c}\text { Moderate Turbulence } \\
\varepsilon, \mathrm{m}^{2} / \mathrm{s}^{3}\end{array}$ & $\begin{array}{c}\text { Severe Turbulence } \\
\varepsilon, \mathrm{m}^{2} / \mathrm{s}^{3}\end{array}$ & $\begin{array}{c}\text { Normalized } \\
\text { variation, } \varepsilon_{\mathrm{D}}\end{array}$ \\
\hline 1 & $5.53 \mathrm{E}-05$ & 0.0506 & 2.09 & 2.77 \\
2 & $5.10 \mathrm{E}-05$ & 0.0467 & 2.03 & 2.56 \\
4 & $7.21 \mathrm{E}-05$ & 0.0765 & 2.19 & 3.61 \\
6 & $8.34 \mathrm{E}-05$ & 0.0871 & 3.31 & 4.81 \\
8 & $9.59 \mathrm{E}-05$ & 0.0895 & 3.94 & 4.07 \\
10 & $8.11 \mathrm{E}-05$ & 0.0845 & 3.50 & 4.08 \\
12 & $8.13 \mathrm{E}-05$ & 0.0784 & 2.56 & 2.93 \\
14 & $5.84 \mathrm{E}-05$ & 0.0598 & 0.34 & 1.91 \\
16 & $3.81 \mathrm{E}-05$ & 0.0400 & 0.13 & 0.00 \\
18 & 0.0202 & 0.06 & 0.77 \\
20 & $1.32 \mathrm{E}-05$ & 0.0125 & 0.06 & 0.83 \\
25 & $1.54 \mathrm{E}-05$ & 0.0155 & 0.06 & \\
30 & $1.66 \mathrm{E}-05$ & 0.0170 & & \\
\hline
\end{tabular}


Table 3. $\varepsilon$ at regular latitude and longitude intervals (for proper scaling multiply values by $10^{-5} \mathrm{~m}^{2} \mathrm{~s}^{-3}$ ).

\begin{tabular}{|c|c|c|c|c|c|c|c|c|c|c|c|c|c|}
\hline $\begin{array}{l}\text { Longitude } \\
\text { (east), deg }\end{array}$ & 80 & 70 & 60 & 50 & 40 & 30 & 20 & 10 & 0 & -10 & -20 & -30 & -40 \\
\hline 100 & 0.8 & 2.1 & 2.1 & 2.1 & 2.1 & 2.1 & 2.1 & 2 & 1.5 & 1 & 0.3 & 0.3 & 0.3 \\
\hline 120 & 1 & 2.1 & 2.1 & 2.1 & 2.1 & 2.1 & 4 & 4 & 2 & 1.5 & 1 & 0.3 & 0.3 \\
\hline 140 & 1 & 2.1 & 6 & 6 & 8 & 6 & 3 & 4 & 6 & 3 & 2.1 & 0.3 & 0.3 \\
\hline 160 & 0.3 & 2.1 & 2.1 & 3 & 1.9 & 1.5 & 1.4 & 2 & 2 & 2 & 1.6 & 1 & 0.3 \\
\hline 180 & 0.3 & 1 & 2.2 & 2.5 & 1 & 1.3 & 1.4 & 1.5 & 2 & 1.8 & 1 & 0.3 & 0.3 \\
\hline-160 & 0.3 & 2.1 & 2.1 & 1.5 & 1.3 & 1.8 & 2 & 1 & 2 & 1 & 1 & 0.3 & 0.3 \\
\hline-140 & 0.3 & 2.1 & 2.1 & 1 & 1.2 & 1.5 & 1.5 & 1.5 & 2 & 1 & 1 & 0.3 & 0.3 \\
\hline-120 & 1 & 2.1 & 2.1 & 2.1 & 2.1 & 1 & 1 & 1.8 & 2 & 1 & 1 & 0.3 & 0.3 \\
\hline-100 & 1 & 2.1 & 2.1 & 2.1 & 2.1 & 2.1 & 1 & 2 & 2 & 1 & 1 & 0.3 & 0.3 \\
\hline-80 & 1 & 2.1 & 2.1 & 2.1 & 2.1 & 2 & 2 & 2 & 2 & 1.5 & 1 & 0.3 & 0.3 \\
\hline-60 & 1 & 0.3 & 0.3 & 2 & 1.3 & 1 & 1 & 2 & 2.1 & 2.1 & 2.1 & 0.3 & 0.3 \\
\hline 40 & 1 & 2.1 & 2 & 1 & 2 & 1.5 & 1.2 & 2 & 2 & 1.5 & 1 & 0.3 & 0.3 \\
\hline-20 & 2 & 2 & 1.8 & 1.2 & 1.5 & 1.2 & 1.2 & 2 & 1.8 & 1 & 1 & 0.3 & 0.3 \\
\hline 0 & 1.5 & 1.5 & 2 & 2 & 1 & 2.1 & 2.1 & 2.1 & 1.5 & 1 & 0.3 & 0.3 & 0.3 \\
\hline 20 & 1 & 2 & 2.1 & 2.1 & 2.1 & 2.1 & 2.1 & 2.1 & 2.1 & 2.1 & 2.1 & 2.1 & 0.3 \\
\hline 40 & 1.5 & 1.5 & 2.1 & 2.1 & 2.1 & 2.1 & 2.1 & 2.1 & 2.1 & 0.3 & 0.3 & 0.3 & 0.3 \\
\hline 60 & 1.5 & 1.5 & 2.1 & 2.1 & 2.1 & 2.1 & 2 & 1.5 & 1 & 0.3 & 0.3 & 0.3 & 0.3 \\
\hline 80 & 1.5 & 2.1 & 2.1 & 2.1 & 2.1 & 2.1 & 2.1 & 1.5 & 1 & 0.3 & 0.3 & 0.3 & 0.3 \\
\hline
\end{tabular}

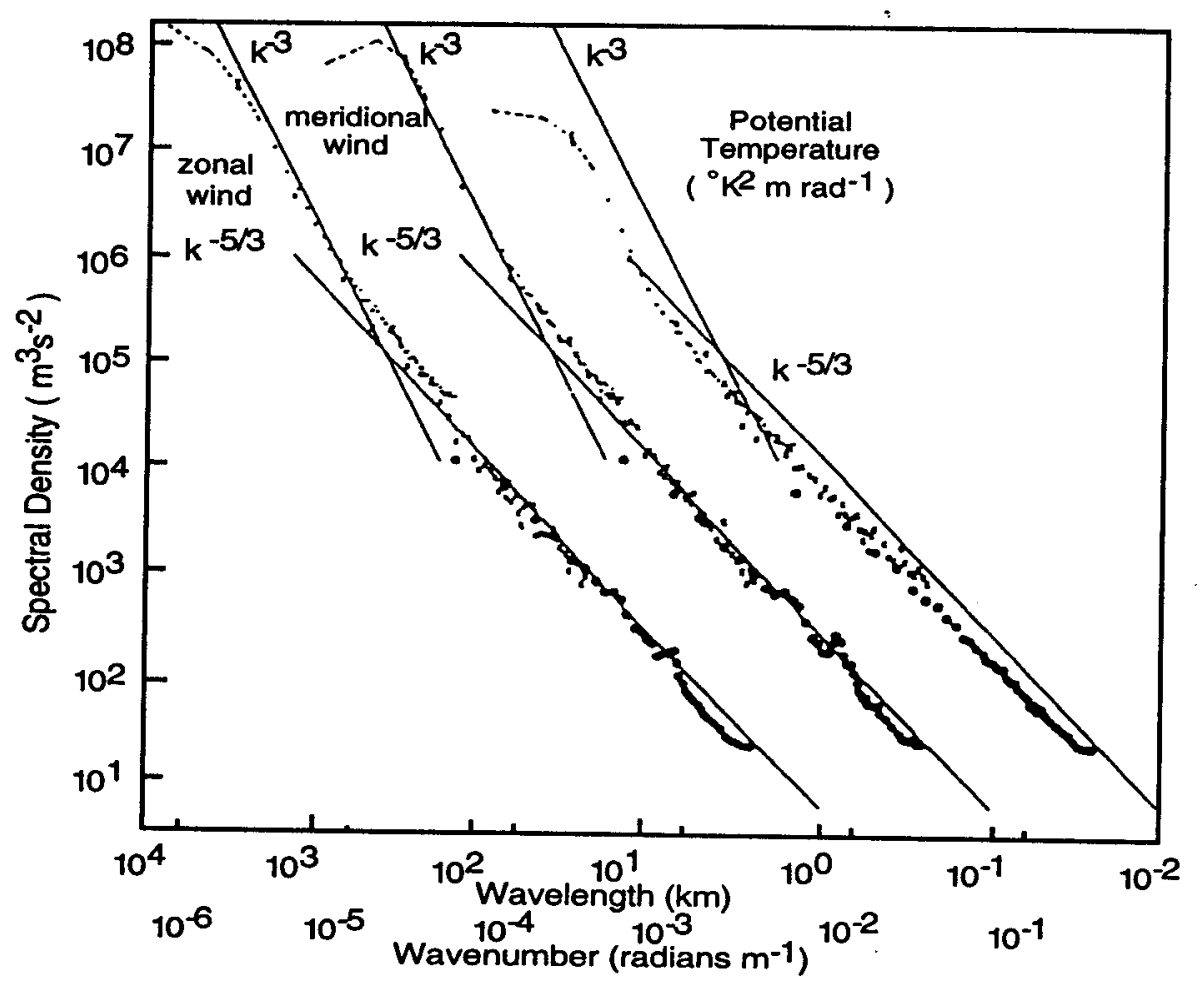

Figure 1. Wind and Potential Temperature Spectra as reported by Nastrom and Gage (1981). Note: for clarity, the meridional wind and temperature spectra have been shifted one and two decades to the right, respectively. 

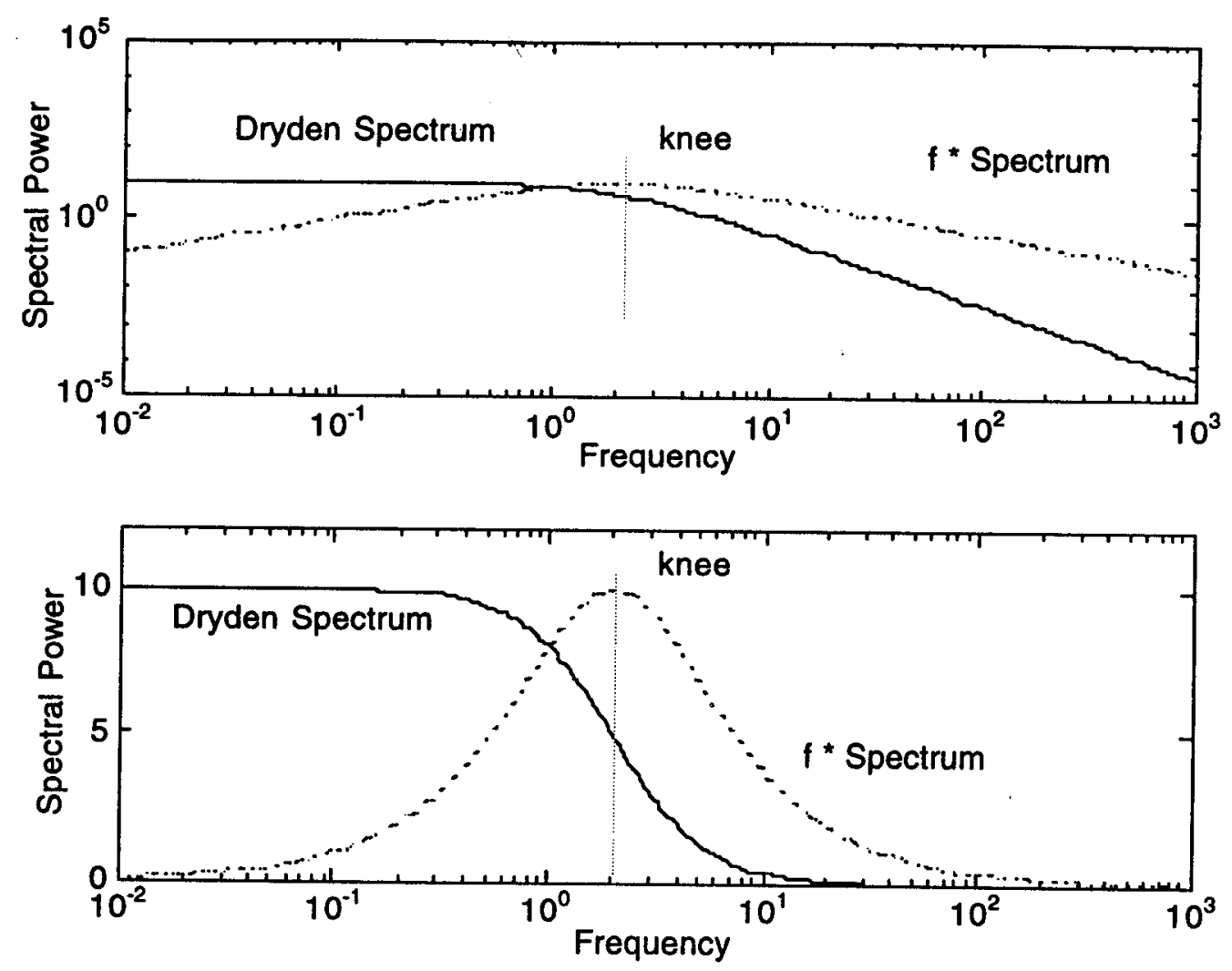

Figure 2. -Log and semi-log plots of Dryden Spectrum showing 'knee' frequency (rad./m).

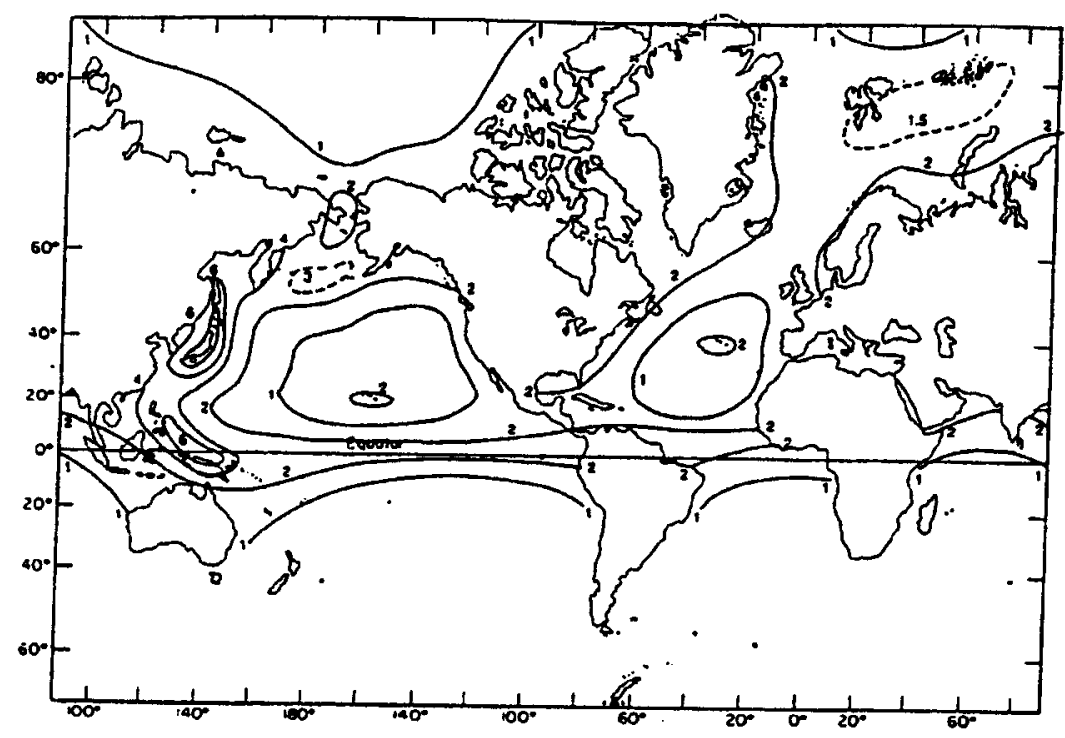

Figure 3.- Lower stratosphere, annual mean, eddy dissipation rate, $\times 10^{5}$ $\mathrm{m}^{2} / \mathrm{s}^{3}$, from reference 7 . 


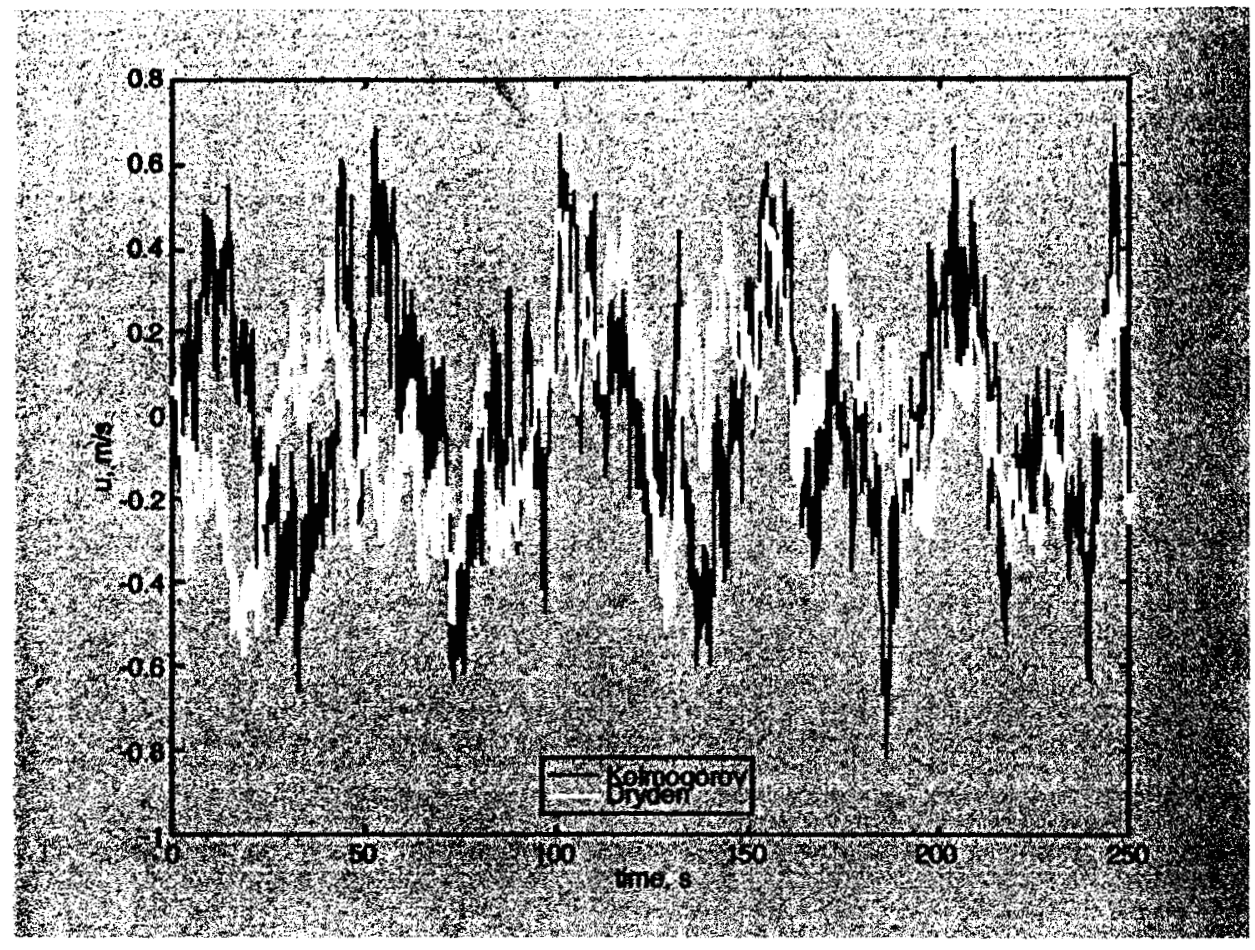

Figure 4.- Comparison of longitudinal turbulence time histories evolved by the Dryden filter method $\left(\sigma=0.22 \mathrm{~m} / \mathrm{s}\right.$ and $\left.L_{u}=5 \mathrm{~km}\right)$ and the Tank model $\left(\mathrm{f}_{1}=.0125 \mathrm{~Hz}, \mathrm{f}_{2}=5 \mathrm{~Hz}, \varepsilon=2 \times 10^{-5} \mathrm{~m}^{2} / \mathrm{s}^{3}\right)$.

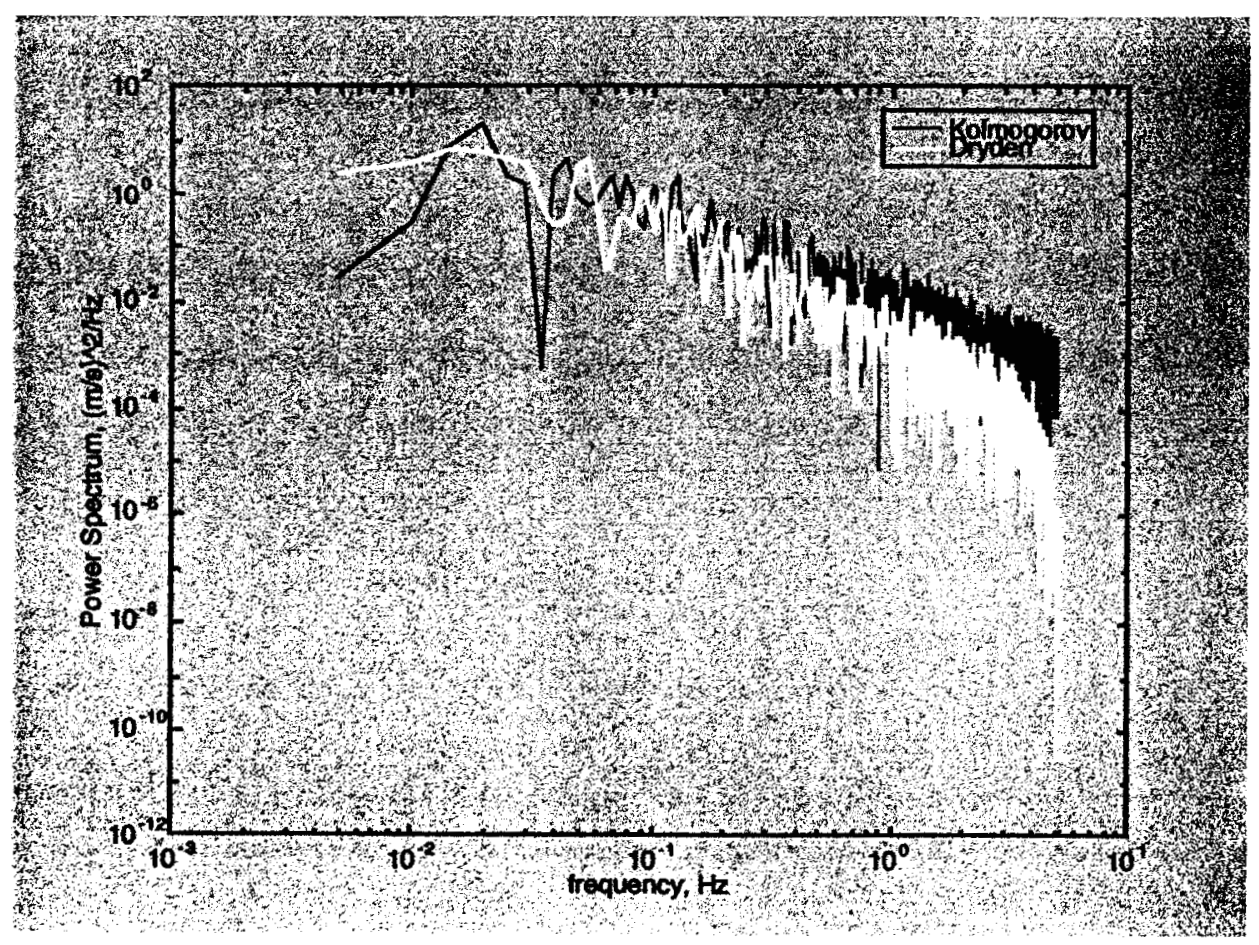

Figure 5.- Power spectra of time histories shown in Figure 4. 


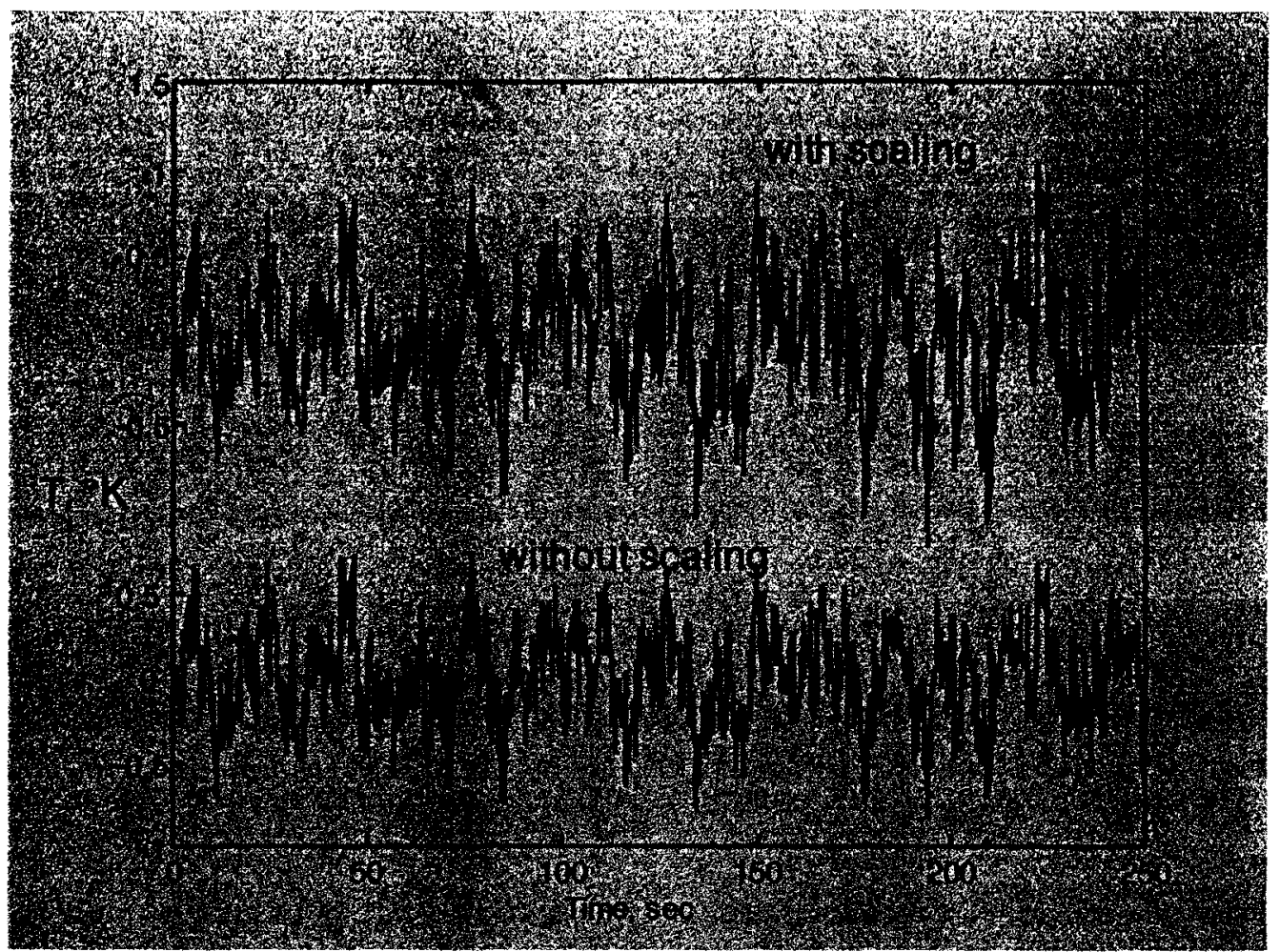

Figure 6.- Temperature histories with and without $\varepsilon$ scaling.
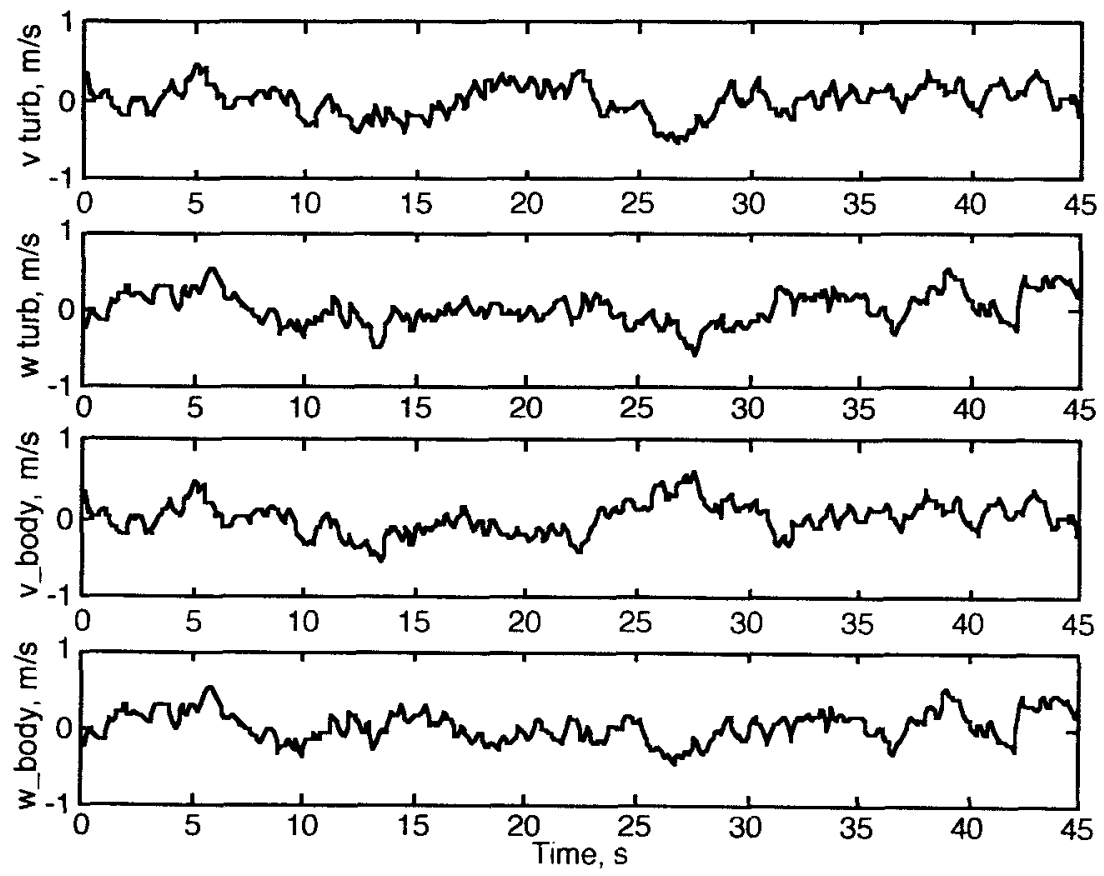

Figure 7.- Comparison of transverse turbulence in turbulence and body axes during $\mathbf{3 6 0}$ degree roll. 


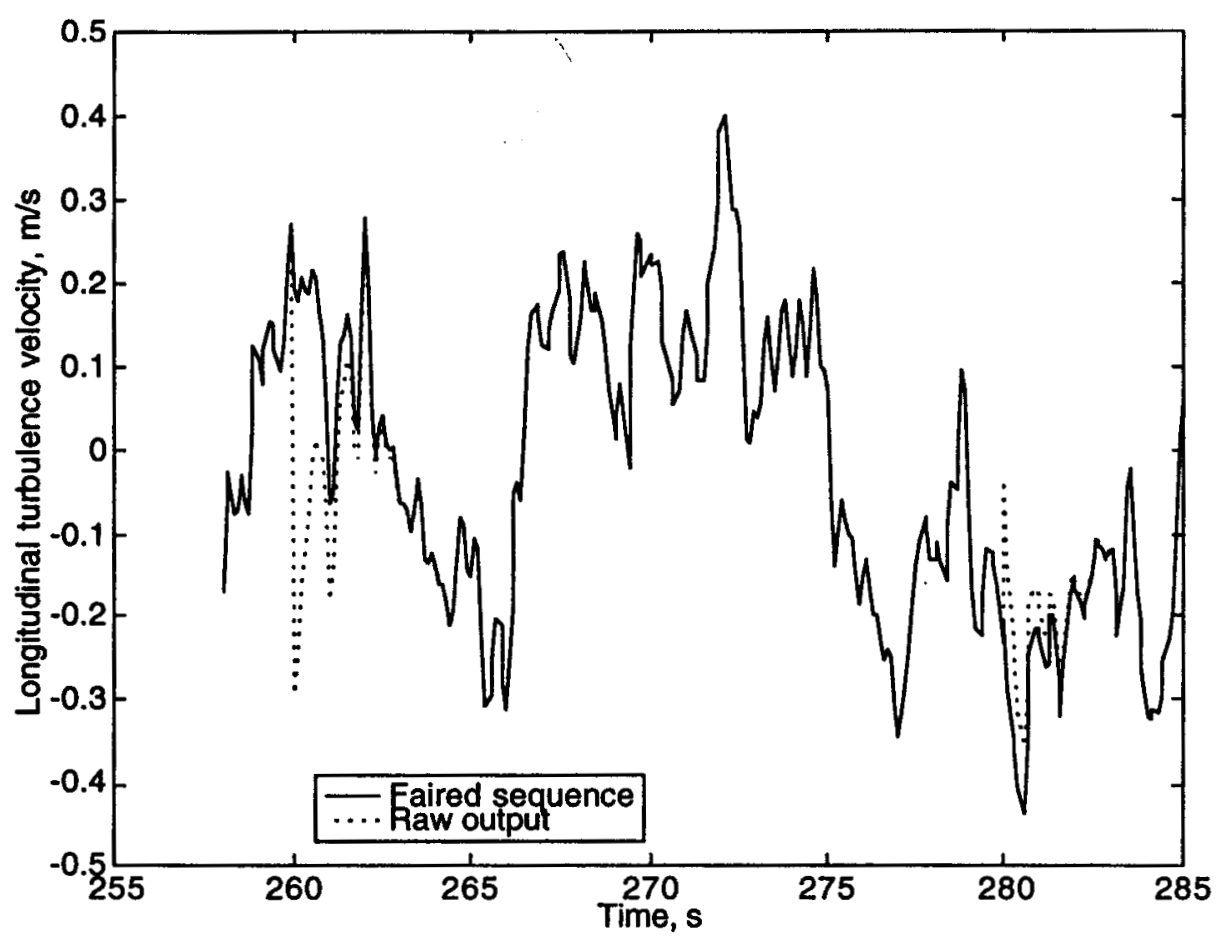

Figure 8.- Longitudinal turbulence time history showing the effect of 'fairing' between segments. 